\title{
Investigation of the solubility and dispersion degree of calf skin collagen in ionic liquids
}

Sicong Liu ${ }^{1,2}$, Qian Li $i^{1}$ and Guoying $\mathrm{Li}^{1,2^{*}}$ (D)

\begin{abstract}
The dissolution of collagen in ionic liquids (ILS) was highly dependent on the polarity of ILS, which was influenced by their sorts and concentrations. Herein, the solubility and dispersion degree of collagen in two sorts of ILS, namely 1-ethyl-methylimidazolium tetrafluoroborate ([EMIM] [BF $]$ ) with low polarity and 1-ethyl-3methylimidazolium acetate ([EMIM][AC]) with high polarity in a concentration range from $10 \%$ to $70 \%$ at $10^{\circ} \mathrm{C}$ were investigated. When $150 \mathrm{mg}$ of collagen was added to $30 \mathrm{mg}$ of ILs, the minimum soluble collagen concentration was $0.02 \mathrm{mg} / \mathrm{mL}$ in $70 \%\left[\mathrm{EMIM}_{[}\left[\mathrm{BF}_{4}\right.\right.$ ] with lowest polarity and the maximum was $3.57 \mathrm{mg} / \mathrm{mL}$ in $70 \%$ [EMIM] [Ac] with highest polarity, which indicates that soluble collagen and insoluble collagen fibers were both present. For insoluble collagens, differential scanning calorimetry showed that the thermal-stability was weakened when increasing the ILs concentration and polarity, and the fiber arrangement was looser with a more uniform lyophilized structure, observed by atomic force microscopy and scanning electron microscopy. For soluble collagens, electrophoresis patterns and Fourier transform infrared spectroscopy showed that no polypeptide chain degradation occurred during dissolution, but the thermal denaturation temperature decreased by $0.26{ }^{\circ} \mathrm{C} \sim 7.63^{\circ} \mathrm{C}$ with the increase of ILs concentrations, measured by ultra-sensitive differential scanning calorimetry. Moreover, the aggregation of collagen molecules was reduced when ILs polarity was increased as determined by fluorescence measurements and dynamic light scattering, which resulted in an increased loose fiber arrangement observed by atomic force microscopy. If the structural integrity of collagen needs to be retained, then the ILs sorts and concentrations should be considered.
\end{abstract}

Keywords: Ionic liquids concentrations, Collagen solubility, Structural integrity, Dispersion degree, Aggregation state

\section{Introduction}

Ionic liquids (ILs), which are known as "supramolecular solvents", have appeared as novel solvents that permit the compounding of a series of conventional natural biomasses that are not easily soluble. ILs are molten salts that are composed of bulky organic cations and small inorganic anions $[1,2]$. As promising alternatives to conventional organic solvents, ILs possess many unmatched favorable properties, such as miscibility with water and organic solvents, high tunability, low or negligible vapor

\footnotetext{
*Correspondence: liguoyings@163.com

'National Engineering Laboratory for Clean Technology of Leather Manufacture, Sichuan University, Chengdu 610065, People's Republic of China

${ }^{2}$ Key Laboratory of Leather Chemistry and Engineering (Ministry of Education), Sichuan University, Chengdu 610065, People's Republic of China
}

pressure, and thus, they are classified as "green solvents" [3-5]. Recently, progress has been rapid in the application of ILs as a novel solvent type. Fukaya et al. [6] found that 1-ethyl-3-methyl imidazolidic phosphonate ionic liquid had an excellent solubility in cellulose, and the dissolution rate of cellulose was $10 \%$ at $30 \mathrm{~min}$ and $45^{\circ} \mathrm{C}$. The solubility of chitosan with a molecular weight of $9.7 \mathrm{kDa}$ could reach $12 \%$ in [C4mim] [CH3COO] at $110^{\circ} \mathrm{C}$ as reported by Wu et al. [7] Philips et al. [8] studied the solubility of silk fibroin in various ILs, and found that $[\mathrm{BMIM}] \mathrm{Cl}$ had the highest solubility for protein fibers.

Because many reports have verified the possibility of dissolving natural polymer, such as cellulose, chitosan, and biological protein with ILs, researchers started to consider the application of the high polarity ILs in 
collagen dissolution. Collagen, as the most abundant fibrous protein in connective tissues of land-based mammals (e.g. calf skin) which are common raw materials in leather industry, has been widely applied in food, pharmaceutical, biochemistry and cosmetic products, because of its unusual combination of good biocompatibility, low antigenicity and controlled biodegradability [9-12]. These great biological properties of collagen are ascribed mostly to its unique triple helix conformation, which is stabilized by hydrogen bonds [13-15]. However, the molecular weight of $\sim 300 \mathrm{kDa}$ and the large numbers of hydrogen bonds, electrovalent bonds, hydrophobic bonds, and van der Waals forces prevent collagen from being dissolved in general solvents, but it is highly desirable that ILs provide a high transformation and ability to utilize collagen.

The applications of ILs in related collagen research have focused mainly on a solvent medium of blend [1618], pretreatment solvent of collagen extraction [10, 19], or conditions of collagen dissolution and changes in regenerated-collagen properties [20-22]. Wang et al. [16] studied the dissolution performance of white-hidepowder from leather-making in $\left[\mathrm{C}_{6} \mathrm{O}_{2}(\mathrm{mim})_{2}\right][\mathrm{Br}]_{2}$, and found that when the white hide powder solubility reached $8 \%$, the dissolving time was $55 \mathrm{~min}$ at $120^{\circ} \mathrm{C}$. The triple helix structure of white-hide-powder was destroyed partially during $\left[\mathrm{C}_{6} \mathrm{O}_{2}(\mathrm{mim})_{2}\right][\mathrm{Br}]_{2}$ dissolution. As Meng et al. [21] reported, native skin collagen fibers could dissolve in [BMIM] Cl at $100^{\circ} \mathrm{C}$ for $6 \mathrm{~h}$, but the triple helix structure of collagen was partially disturbed during the dissolution and regeneration. The collagen materials used in these studies were usually not highly purified collagen. Hu et al. [22] dissolved collagen in [EMIM] [Ac] with a series of sodium salts at different temperatures $(25,30,35,40$, and $45^{\circ} \mathrm{C}$ ) to evaluate [EMIM] [Ac]/sodium-salt systems as suitable solvents for collagen. The [EMIM][Ac] solvent system broke most of the hydrogen bonds between the amino hydroxyl and ester oxygen of collagen. A higher temperature appeared to promote the intermolecular distance of collagen significantly and allowed ILs to destroy hydrogen bonds more easily. To prepare the collagen solution efficiently, these studies usually dissolved collagen in ILs at a higher temperature, which was easy to damage the triplehelix structure of collagen. Also, since complete dissolution of collagen in ILs was not achieved, the insoluble part of collagen had not been thoroughly studied. Furthermore, it should be noted that both the sorts and concentrations of ILs, which are closely related to their polarity, have great influences on the properties of collagen [10, 19, 23-28]. For example, the differences in composition of imidazolium-based ILs endowed by varying anions with different polarity namely acetate, hydrogen sulfate, dicyanamide, dimethyl phosphate, dihydrogen phosphate and sulfate, had resulted in different destabilizing effects on the triple helical structure of collagen [25]; a notable decreasing trend in denaturation temperature of collagen fibers was discovered with the increase of ILs concentration from $0.1 \%$ to $10 \%(w / v)$ [10]. Therefore, it will be of importance and significance to study how the ILs sorts and concentrations would affect the structure and properties of collagen in the dissolution process. Moreover, the dispersion degree is a core property of collagen, affecting its functional performances as biomaterials, for instance, the viscoelastic behavior originated by the aggregation of collagen molecules [22]. Thus, the dispersion degree of collagen after the dissolving process should also be investigated.

Herein, we controlled the collagen dissolution at a lower temperature with more safety $\left(\sim 10^{\circ} \mathrm{C}\right)$ and investigated the solubility and the dispersion degree of collagen fibrils in two types of 1-ethyl-methylimidazolium ([EMIM]) based ILs containing different anions ([EMIM] tetrafluoroborate $\left([\mathrm{EMIM}]\left[\mathrm{BF}_{4}\right]\right)$ and $[\mathrm{EMIM}]$ acetate $([\mathrm{EMIM}][\mathrm{Ac}])$, which are liquid state at room temperature), the ions of which exhibited good biocompatibility and mild polarity [22, 29]. [EMIM] [Ac] was used in a concentration range of $10 \%-$ $70 \%$, while $[\mathrm{EMIM}]\left[\mathrm{BF}_{4}\right]$ was used solely in $70 \%$, owing to its low polarity. The solubility of collagen in ILs was tested, and insoluble as well as soluble collagens were examined separately to determine their structural integrity and dispersion degree. The obtained results will help to understand the effect of ILs on the solubility and dispersion degree of collagen while maintaining its structural integrity and provide guidance for the application of ILs in collagen research.

\section{Experimental}

\subsection{Materials}

Native collagen used in this study was extracted from calf skin by $0.5 \mathrm{M}$ acetic acid that contained 3\% pepsin according to the method of Zhang et al. with a slight modification [30]. The ionic liquids (ILs), 1-ethyl-methylimidazolium tetrafluoroborate ([EMIM] $\left.\left[\mathrm{BF}_{4}\right]\right)$ and 1-ethyl-3-methylimidazolium acetate ([EMIM][Ac]) were purchased from Lanzhou Institute of Chemical Physics (Chinese Academy of Sciences, Lanzhou, China) with high purification $(\geq 99 \%)$. [EMIM] [Ac] was diluted in deionized water at concentrations of $30 \%, 50 \%$, and $70 \%(\mathrm{w} / \mathrm{w})$ respectively, while $[\mathrm{EMIM}]\left[\mathrm{BF}_{4}\right]$ was diluted in deionized water solely at $70 \%$ (w/w). 8-Anilino-1-naphthalenesulfonate (ANS) was from Sigma Chemicals (Sigma-Aldrich, Munich, Germany). Other chemicals were from Chengdu Kelong Industrial (Chengdu, China).

\subsection{Processing collagen with ILs}

Lyophilized collagen $(150 \mathrm{mg})$ was added to a beaker with $30 \mathrm{~g}$ of [EMIM] $\left[\mathrm{BF}_{4}\right](70 \%)$ or different concentrations of [EMIM] $\mathrm{Ac}](10 \%, 30 \%, 50 \%, 70 \%)$ to achieve an 
ideal concentration of $5 \mathrm{mg} / \mathrm{mL}$. All mixtures were stirred ceaselessly by magnetic stirrer for $48 \mathrm{~h}$ in water bath at $10{ }^{\circ} \mathrm{C}$, and then centrifuged at $7900 \mathrm{rpm}$ for 10 min by using a high-speed freezer centrifuge. The insoluble collagen fibrils (Col/ILs-fibrils10\%, Col/ILs-fibrils30\%, Col/ILs-fibrils50\%, Col/ILs-fibrils70\%, Col/ILsfibrils70\%B) were washed with distilled water several times, dissolved in $0.5 \mathrm{M}$ acetic acid, and dialyzed against $0.1 \mathrm{M}$ acetic acid for 3 days to remove ILs. The supernatant, which is the soluble collagen (Col/ILs-soluble10\%, Col/ILs-soluble30\%, Col/ILs-soluble50\%, Col/ ILs-soluble70\%) was reserved for the following measurements. The obtained specimen was lyophilized by freeze drying and preserved in a desiccator before used.

\subsection{Collagen solubility in ILs}

The solubility of collagen (Col/ILs-fibrils70\%B, Col/ILssoluble10\%, Col/ILs-soluble30\%, Col/ILs-soluble50\%, Col/ILs-soluble70\%) were measured using a method recommended by Li et al. [31] The soluble collagen was diluted with $6 \mathrm{M} \mathrm{HCl}$ at a 1:5 ratio and mixed evenly. Briefly, diluents were added to ampere bottles three times and then sealed for hydrolysis for $24 \mathrm{~h}$ at $110^{\circ} \mathrm{C}$. The hydrolysates were transferred to a rotary evaporator and distilled in a vacuum with water bath at $60^{\circ} \mathrm{C}$. After evaporation, the solid was transferred to $25-\mathrm{mL}$ volumetric flasks with citrate-acetate-buffer ( $\mathrm{pH} 6)$. The sample solutions $(300 \mu \mathrm{L})$ were mixed with $600 \mu \mathrm{L}$ of isopropanol and $300 \mu \mathrm{L}$ of liquid A (7\% chloramine $\mathrm{T}$ solution:pH 6 citrate-acetate-buffer $=1: 4)$. The absorbance was then measured at $558 \mathrm{~nm}$, using an ultraviolet and visible spectrophotometer (Lambda 25, PerkinElmer, USA). The values of soluble collagen concentration (C) were calculated from:

$$
\mathrm{C}=\frac{\mathrm{y}-0.0042}{0.1687} \times \mathrm{v} \times \mathrm{A} \div 1000
$$

where $\mathrm{y}$ is the absorbance, $\mathrm{v}$ is the volume dilution ratio, and $\mathrm{A}$ is the conversion coefficient of hydroxyproline concentration and collagen concentration. The standard curve of hydroxyproline is $y=0.1687 x+0.0042\left(R^{2}=0.999\right)$.

\subsection{DSC of insoluble collagen fibrils}

The thermal stability of the lyophilized Col/ILs-fibrils70\%B, Col/ILs-fibrils10\%， Col/ILs-fibrils30\%， Col/ILs-fibrils50\% and Col/ILs-fibrils70\% was detected by using DSC (Netzsch DSC 200PC, Bayern, Germany). The freeze-dried Col and collagen fibrils $(\sim 3 \mathrm{mg})$ were weighed into aluminum pans and sealed, then scanned in a nitrogen atmosphere from 35 to $90^{\circ} \mathrm{C}$ at $2{ }^{\circ} \mathrm{C} / \mathrm{min}$. The measurements were performed in triplicate.

\subsection{SEM of insoluble collagen fibrils}

The morphologies of the lyophilized Col/ILs-fibrils70\%B, Col/ILs-fibrils10\%, Col/ILs-fibrils30\%, Col/ILs-fibrils50\% and Col/ILs-fibrils70\% were observed by SEM (S-800, HITACHI, Japan) at $20 \mathrm{kV}$. Images of each sample were obtained at different spots to confirm the consistency of the morphology observed.

\subsection{AFM measurements of insoluble and soluble collagen}

The morphologies of the insoluble collagen fibrils (Col/ ILs-fibrils70\%B， Col/ILs-fibrils10\%， Col/ILs-fibrils30\%, Col/ILs-fibrils50\% and Col/ILs-fibrils70\%) and the soluble collagen (Col/ILs-soluble10\%, Col/ILs-soluble30\%, Col/ILs-soluble50\% and Col/ILs-soluble70\%) were observed by AFM with a pinpoint. The insoluble collagen fibrils were dispersed in $0.5 \mathrm{M}$ acetic acid. The soluble collagen was dispersed in corresponding concentrations of [EMIM][Ac]. The corresponding concentration of collagen was used as a reference. $15 \mu \mathrm{L}$ of each diluted samples was dropped on mica and then dried at $30{ }^{\circ} \mathrm{C}$ for 3 days. Particular attention was given to the fact that 1 day after drying, it was necessary to rinse the mica sheets of Col/ILs-soluble10\%, Col/ILs-soluble30\%, Col/ ILs-soluble $50 \%$, and Col/ILs-soluble70\% slowly with distilled water to remove ILs.

\subsection{SDS-PAGE patterns of soluble collagen}

SDS-PAGE was performed according to the method of Laemmli [32] with slight modifications. The lyophilized collagen was dissolved in $0.1 \mathrm{M}$ acetic acid $(\mathrm{Col}) . \mathrm{Col}, \mathrm{Col} /$ ILs-soluble10\%, Col/ILs-soluble30\%, Col/ILs-soluble50\%, and Col/ILs-soluble70\% were mixed with buffer solution and then boiled in water for $5 \mathrm{~min}$. Then, each sample was loaded for each lane and electrophoresed by MiniPROTEAN 3 Cell (Bio-Rad, California, USA). After electrophoresis, the gel was dyed by $0.25 \%$ coomassie brilliant blue R-250 and then destained with 7.5\% acetic acid and $5 \%$ methanol.

\subsection{FTIR of soluble collagen}

All collagen samples were homogeneously triturated with $300 \mathrm{mg}$ potassium bromide $(\mathrm{KBr})$ and the mixtures were made into disks under a pressure of $20 \mathrm{MPa}$. The FTIR spectra were recorded by a Nicolet iS10 spectrometer (Thermo Fisher Scientific, Waltham, MA, USA) at the wavenumbers ranging from 4000 to $500 \mathrm{~cm}^{-1}$ with a resolution of $2 \mathrm{~cm}^{-1}$ at $25^{\circ} \mathrm{C}$.

\subsection{US-DSC of soluble collagen}

The thermal stability of the soluble collagen Col/ILs-soluble10\%, Col/ILs-soluble30\%, Col/ILs-soluble50\%, and Col/ILs-soluble70\% was determined by US-DSC (VP-DSC, Microcal, Northampton, USA) and matching concentrations of [EMIM][Ac] and Col were used as references. All 
specimens were measured at $0.5 \mathrm{mg} / \mathrm{mL}$ and performed in triplicate.

\subsection{Fluorescence measurements of soluble collagen}

Fluorescence measurements of ANS in Col/ILs-soluble $10 \%$, Col/ILs-soluble $30 \%$, Col/ILs-soluble $50 \%$, and Col/ILs-soluble70\% were measured by a Hitachi F-7000 fluorescence spectrophotometer (Hitachi, Tokyo, Japan), as described by Kamyshny et al. [33] with a slight modification. The same concentration of soluble collagen $(0.5 \mathrm{mg} / \mathrm{mL})$ was prepared to evaluate the aggregation status of collagen molecules. Briefly, the ANS probe was prepared in $0.1 \mathrm{~mol} / \mathrm{L}$ sodium phosphate buffer ( $\mathrm{pH} 7.0$ ) to obtain a $400 \mathrm{mmol} / \mathrm{L}$ transparent solution. Then, 10 $\mathrm{mL}$ sample solutions were mixed with $50 \mu \mathrm{L}$ of ANS solution and the mixtures were homogenized. The fluorescence spectrum was recorded at $20^{\circ}$. The excitation wavelength was fixed at $432 \mathrm{~nm}$ and the emission spectra were measured from 450 to $750 \mathrm{~nm}$. The slits were fixed at $5 \mathrm{~nm}$.

\subsection{DLS measurements of soluble collagen}

A DLS technique that uses a Zetasize (Nano-ZS, Malvern Instruments Ltd., Malvern, UK) was employed for the soluble collagen Col/ILs-soluble10\%, Col/ILs-soluble $30 \%$, Col/ILs-soluble $50 \%$, and Col/ILs-soluble70\% to obtain information on the size distribution and average aggregation size. The sample solutions were first diluted to $1.0 \mathrm{mg} / \mathrm{mL}$, then filtered through a $2-\mu \mathrm{m}$ filter (Millipore, Billerica, USA) and measured in a polystyrene cuvette.

\section{Results and discussion}

\subsection{Solubility of collagen in ILs}

The dissolution behavior of collagen in ILs was investigated. Table 1 shows the concentration of soluble collagen in $[\mathrm{EMIM}]\left[\mathrm{BF}_{4}\right]$ and different concentrations of [EMIM][Ac] stirred continually for $48 \mathrm{~h}$ at $10^{\circ} \mathrm{C}$. The solubility of collagen in $[\mathrm{EMIM}]\left[\mathrm{BF}_{4}\right]$ was nearly zero, and the concentration of collagen dissolved in 10\% [EMIM][Ac] was $1.94 \mathrm{mg} / \mathrm{mL}$. When the [EMIM][Ac] concentration was increased from $10 \%$ to $70 \%$, the concentration of collagen increased to $3.57 \mathrm{mg} / \mathrm{mL}$, but was still lower than the input. This proved that even in $70 \%$

Table 1 The concentration of soluble collagen in different ILS and concentrations

\begin{tabular}{ll}
\hline ILs and concentrations & $\begin{array}{l}\text { concentration of soluble } \\
\text { collagen }(\mathrm{mg} / \mathrm{mL})\end{array}$ \\
\hline $70 \%[E M I M]\left[\mathrm{BF}_{4}\right]$ & 0.02 \\
$10 \%[\mathrm{EMIM}][\mathrm{Ac}]$ & 1.94 \\
$30 \%[\mathrm{EMIM}][\mathrm{Ac}]$ & 2.61 \\
$50 \%[\mathrm{EMIM}][\mathrm{Ac}]$ & 3.25 \\
$70 \%[\mathrm{EMIM}][\mathrm{Ac}]$ & 3.57 \\
\hline
\end{tabular}

[EMIM][Ac], the collagen did not achieve complete dissolution. A phenomenon of partial dissolution was resulted with the soluble collagen and insoluble collagen fibers, and the solubility of collagen was affected markedly by ILs sorts and concentrations. This could be likely due to the disrupting of intermolecular hydrogen bonds and ionic bonds in collagen, not only by the bulky [EMIM] $^{+}$that have the capability to donate proton, but also by the proton-accepting anions including $\mathrm{Ac}^{-}$and $\mathrm{BF}_{4}^{-}$. Because of the low polarity and minor protonaccepting ability of $\mathrm{BF}_{4}{ }^{-}$, there was no dissolution of collagen in $[\mathrm{EMIM}]\left[\mathrm{BF}_{4}\right]$. For $[\mathrm{EMIM}][\mathrm{Ac}]$, with the increase of concentration and polarity, the degree of rupturing of hydrogen bonds was deeper, resulting in the increase of solubility. The partial dissolution of collagen led to two forms of collagen in ILs, namely, soluble collagen and insoluble collagen fibrils.

\subsection{Thermal analysis of insoluble collagen fibrils}

The denaturation temperature $\left(\mathrm{T}_{\mathrm{d}}\right)$ of collagen, which is related to the destruction of the native triple helix structure, can be determined by the endothermal peak of DSC $[34,35]$. The thermal transition curves of lyophilized $\mathrm{Col}$ and insoluble collagen fibrils Col/ILs-fibrils70\%B, Col/ ILs-fibrils10\%, Col/ILs-fibrils30\%, Col/ILs-fibrils50\% and Col/ILs-fibrils70\% are shown in Fig. 1. It appears that the $\mathrm{T}_{\mathrm{d}}$ value of Col was $61.9^{\circ} \mathrm{C}$. But the $\mathrm{T}_{\mathrm{d}}$ values of insoluble collagen fibrils were $61.2^{\circ} \mathrm{C}(70 \% \mathrm{~B}), 58.4{ }^{\circ} \mathrm{C}(10 \%), 57.5^{\circ} \mathrm{C}$ (30\%), $55.4^{\circ} \mathrm{C}(50 \%)$, and $50.6^{\circ} \mathrm{C}(70 \%)$, which were lower than that of $\mathrm{Col}$, and which suggested that the insoluble collagen fibrils displayed a weaker thermal stability than the native ones. For Col/ILs-fibrils70\%B derived from $[\mathrm{EMIM}]\left[\mathrm{BF}_{4}\right]$, the decrease was minor. On the contrary, collagen derived from [EMIM][Ac] faced a more

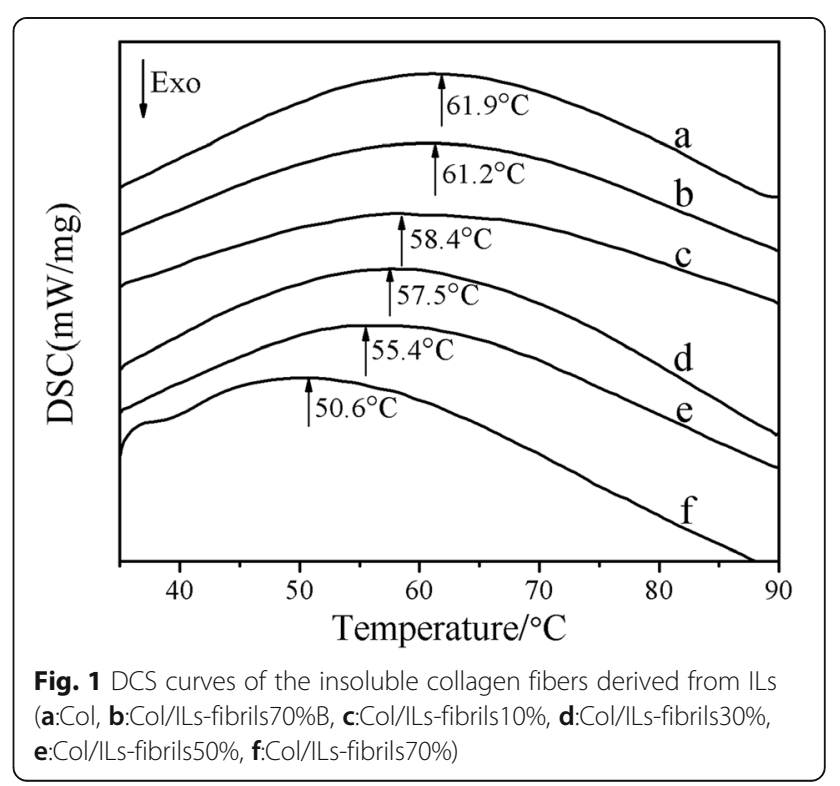


significant decrease with the increase of ILs concentration. The downward tendency of denaturation temperature may be related to the effect of ILs on the fracture of collagen hydrogen bonds. Hu et al. [22] reported that during the dissolving process of collagen in [EMIM][Ac], firstly [EMIM][Ac] will destroy hydrogen bonds between intramolecules or intermolecules; then, after removing [EMI$\mathrm{M}][\mathrm{Ac}]$, some of the hydrogen bonds will be reestablished. Thus, the fiber arrangement became looser, followed by the ability of the insoluble collagen fibers to undergo heat denaturation was reduced. The degree of thermal-stability reduction or the degree of collagen dispersion was correlated with the different capability of ILs destroying hydrogen bonds orientated by polarity, more specifically, their sorts and concentrations.

\subsection{SEM images of insoluble collagen fibrils}

The lyophilized insoluble collagen fibrils Col/ILsfibrils70\%B, Col/ILs-fibrils10\%, Col/ILs-fibrils30\%, Col/ ILs-fibrils50\% and Col/ILs-fibrils70\% were used to obtain morphologies via SEM (Fig. 2). The surface micromorphology of lyophilized collagen was homogeneous with a similar sheet structure and connected pores. The insoluble collagen fibrils retained a relatively homogeneous pore structure, but the surface morphology appeared to be looser, and its pore size was larger than that of native collagen. Moreover, the fibers arrangement became looser, initiated by the destruction of hydrogen bonds as previously discussed. However, the degree of dispersion degree varied in $[\mathrm{EMIM}]\left[\mathrm{BF}_{4}\right]$ and different concentrations of [EMIM][Ac]. Collagen fibers derived from $[\mathrm{EMIM}]\left[\mathrm{BF}_{4}\right]$ were slightly looser than the native ones, while fibers treated by [EMIM][Ac] were much more sparsely distributed with the increase of ILs concentrations. Such differences might be attributed to the lyotropic action and reorientation of the hydration network of collagen induced by ILs with different ions and polarity $[10,23]$. The high polarity can reduce the water associated with collagen, lower its viscosity and consequently increase the mobility of collagen fibers and become looser.

\subsection{AFM images of insoluble collagen fibrils}

Morphological changes of the insoluble collagen fibril molecules (Col/ILs-fibrils70\%B, Col/ILs-fibrils10\%, Col/ ILs-fibrils30\%, Col/ILs-fibrils50\% and Col/ILs-fibrils70\%) were observed by AFM in Fig. 3. This AFM examination suggests that the native collagen exhibited a representative fibrillar structure with fibers entangled and overlapped. The fibril arrangement of the collagen fibers derived from [EMIM] $\left[\mathrm{BF}_{4}\right]$ was a little looser than the native one, while that of [EMIM][Ac] was much looser and presented a more sparse distribution of fiber network in the high concentrated samples. Although the partial interruption of the hydrogen bonds did not make the insoluble collagen fibrils achieve dissolution, the fiber arrangement became looser, and the porosity improved with the increase of ILs polarity and concentration. Overall, these changes in molecular morphologies
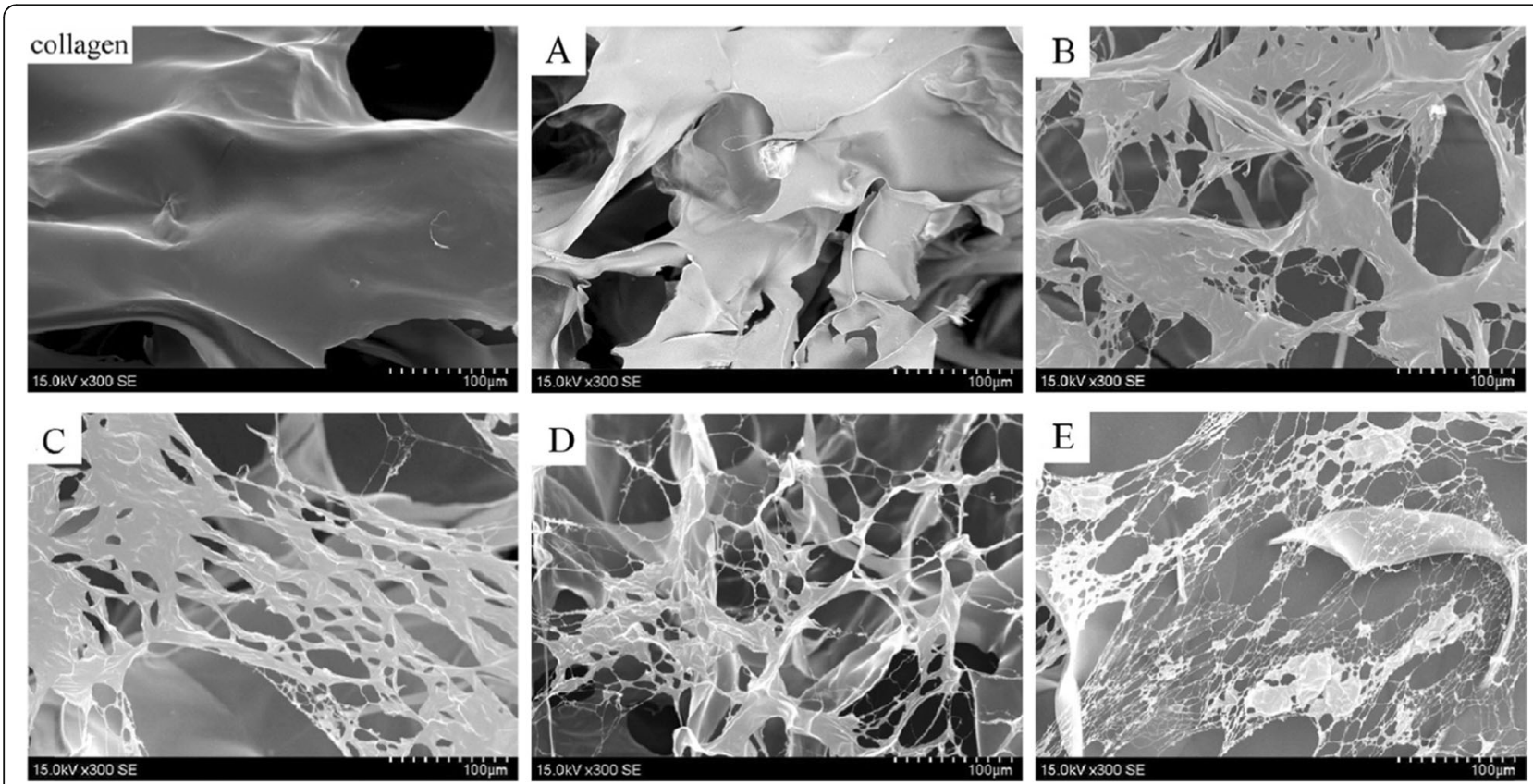

Fig. 2 SEM images of the native collagen and the insoluble collagen fibers derived from ILs: a Col//Ls-fibrils70\%B. b Col//Ls-fibrils10\%. c Col//Lsfibrils30\%. d Col/ILs-fibrils50\%. e Col/LLs-fibrils70\% 


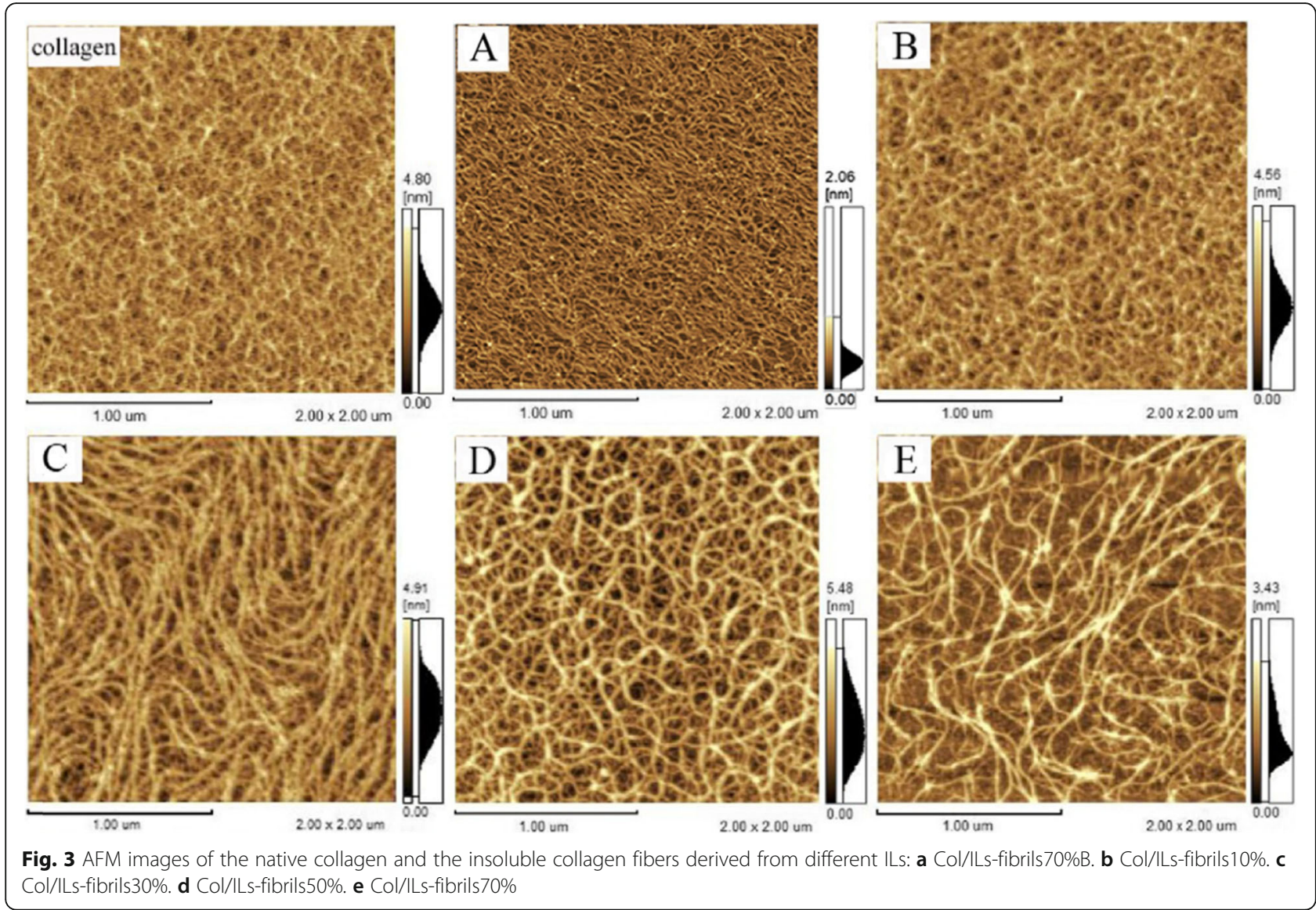

observed by AFM agree well with the surface morphologies aforementioned changes observed by SEM.

\subsection{SDS-PAGE patterns of soluble collagen}

Measurements of electrophoresis analysis were applied to estimate the molecular weight distribution of soluble collagen aggregation within the [EMIM] $[\mathrm{Ac}]$ concentration from $10 \%$ to $70 \%$. Figure 4 displays the SDS-PAGE patterns of Col/ILs-soluble10\%, Col/ILs-soluble30\%, Col/ILssoluble $50 \%$, and Col/ILs-soluble70\%, with $\mathrm{Col}$ as a comparison. All samples are consisted of two typical chains $(\alpha 1$ and $\alpha 2$ ) with molecular weight of approximately $100 \mathrm{kDa}$ and one crosslinked component $\beta$ chain $(\sim 200 \mathrm{kDa})$, which is known as the dimer of $\alpha$ chains. Moreover, the band intensity of $\alpha 1$ was higher than $\alpha 2$ chain, which indicates the collagen might have a $(\alpha 1)_{2} \alpha 2$ structure. These results are in good agreement with the findings that report the characteristic electrophoretic bands of type I collagen. None of the electrophoretic bands existed at lower molecular weights $(<100 \mathrm{kDa})$, indicating no degradation of polypeptide chain happened during dissolution, although some collagen hydrogen bonds had been broken by [EMIM][Ac]. In order to identify the alterations between the native collagen samples and the Col/ILs samples, additional structural analysis should be conducted.

\subsection{FTIR spectra analysis of soluble collagen}

In addition, to further examine the triple helix conformation of collagen, the secondary structure of Col/ILs-soluble10\%, Col/ILs-soluble30\%, Col/ILs-soluble $50 \%$, and $\mathrm{Col} / \mathrm{ILs}$-soluble70\%, with Col as a comparison was examined by FTIR. The spectra of all collagen samples

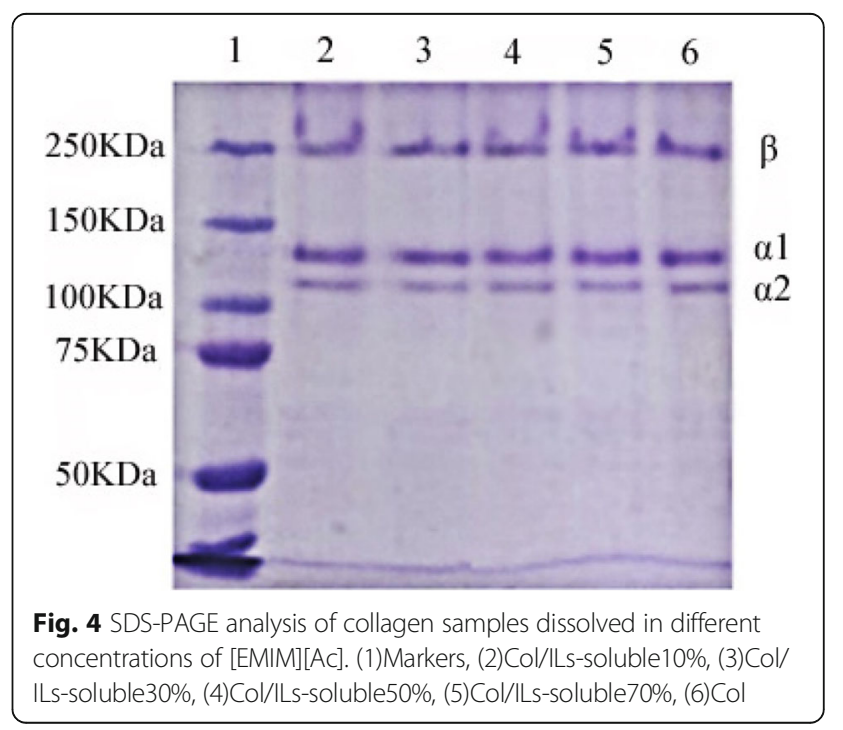


shown in Fig. 5 exhibited the presence of five characteristic FTIR peaks including amide A, B, I, II, and III, which were typical for type I collagen [20]. The amide A peaks of $3400 \mathrm{~cm}^{-1}$ were related to the hydrogen-bonded $-\mathrm{NH}$ groups, while the amide $\mathrm{B}$ bands were found at wavenumbers of $2940 \mathrm{~cm}^{-1}$, reflecting $\mathrm{C}-\mathrm{H}$ stretching [36]. The amide I bands at $1650 \mathrm{~cm}^{-1}$ were attributed to $\mathrm{C}=\mathrm{O}$ stretching vibrations in the polypeptide backbone, and the amide II bands at $1550 \mathrm{~cm}^{-1}$ were initiated from N-H bending and $\mathrm{C}-\mathrm{H}$ stretching. The bands at $1245 \mathrm{~cm}^{-1}$ representing amide III were dominated by the $\mathrm{C}-\mathrm{H}$ vibrations [31]. Moreover, the absorption ratios between the amide III and $1450 \mathrm{~cm}^{-1}$ bands for all collagens were nearly 1.0, unambiguously suggesting that the triplehelical structure of collagen was preserved [22]. Overall, the FTIR analysis supported the structural integrity of collagen, conforming to the above SDS-PAGE results.

\subsection{Thermal analysis of soluble collagen}

The temperature dependence of the specific heat capacity $(\mathrm{Cp})$ of soluble collagen dissolved in different concentrations of [EMIM][Ac] is shown in Fig. 6. The DSC curves show that every collagen samples exhibited a characteristic thermal denaturation peak. The minor endothermic peak $\left(\mathrm{Tm}_{1}\right)$ at low temperature was owing to a breakage of hydrogen bonds in the aggregates of collagen, which was apt to be neglected, and the major endothermic peak $\left(\mathrm{Tm}_{2}\right)$ at high temperature was initiated by the transition of triple helix to the random coil state [37, 38]. Values of $\mathrm{Tm}_{1}$ as well as $\mathrm{Tm}_{2}$ for varying [EMIM][Ac] concentrations are given in Table 2. The $\mathrm{Tm}_{2}$ values of $\mathrm{Col} / \mathrm{ILs}$-soluble $10 \%$ was $42.47^{\circ} \mathrm{C}$, which was close to $42.73^{\circ} \mathrm{C}$ of Col. With [EMIM] [Ac] addition, the values of $\mathrm{Tm}_{2}$ showed a decreasing tendency from 42.47 to $35.10^{\circ} \mathrm{C}$, which demonstrates the decrease of thermal stability from alterations to interactions in collagen molecules. [EMIM] $\mathrm{Ac}]$ exhibited no endothermic peak. For concentrations of 30\% and 50\%, multiple consecutive endothermic peaks exist, which may be related to the different impact of [EMIM][Ac] on the structure of collagen.

Compared with $\mathrm{Col}$, the thermal denaturation temperature of Col/ILs-soluble70\% was reduced by $7.63^{\circ} \mathrm{C}$, which is larger than the decrease of Colregenerated $-35^{\circ} \mathrm{C}$ with $4.1{ }^{\circ} \mathrm{C}$ reported by $\mathrm{Hu}$ et al. [22] This showed the thermal stability decreased more significantly with [EMIM][Ac] concentration. Hydrogen bonds played an important role in the stabilization of triple-helix structure. The interchain hydrogen bonds were mediated by one water molecule, which likely reinforced the triple-helix at regions that lacked proline [39]. [EMIM][Ac] was deduced to break part of the intermolecular hydrogen bonds. Therefore, less water oriented hydrogen bonding in collagen molecules in [EMIM][Ac] were formed because of the increased hydrophobic interaction [40]. Therefore, the stability of the collagen helix weakened as the [EMIM][Ac] concentration increased and a continuous decrease in the values of the $\mathrm{Tm}_{2}$ was

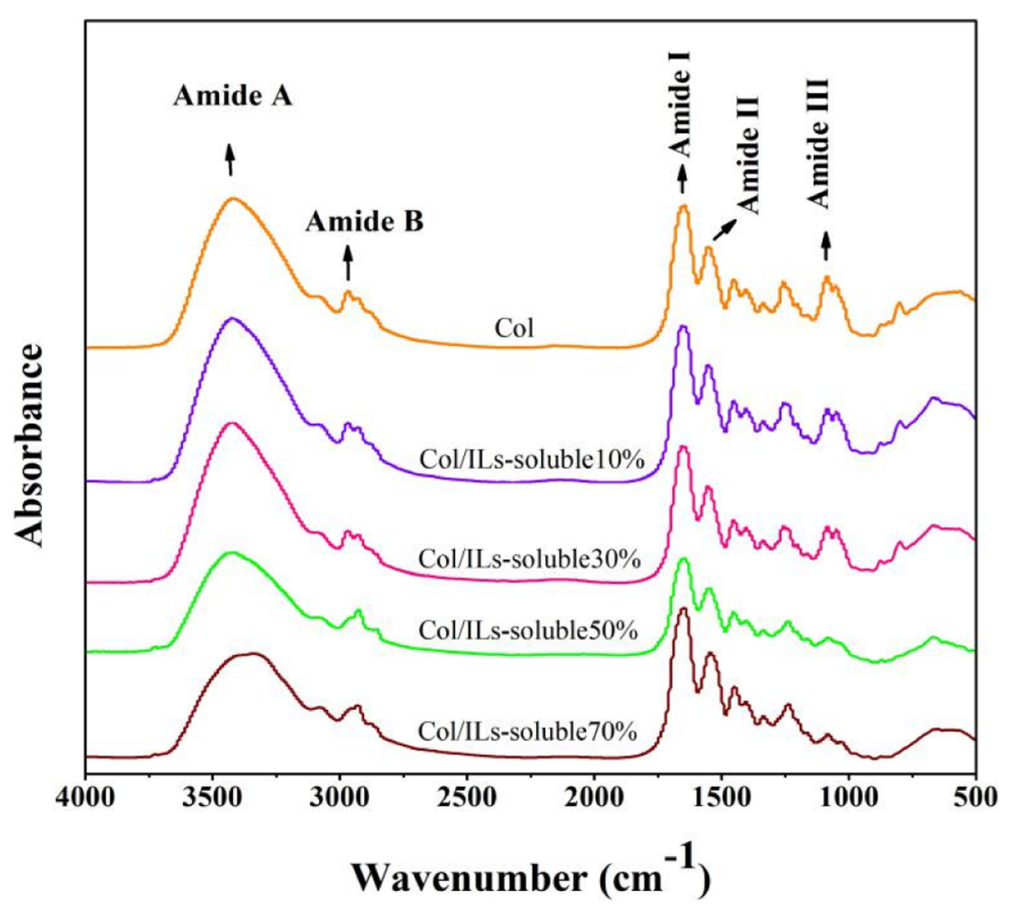

Fig. 5 FT-IR spectra of Col and collagen samples dissolved in different concentrations of [EMIM][AC] 


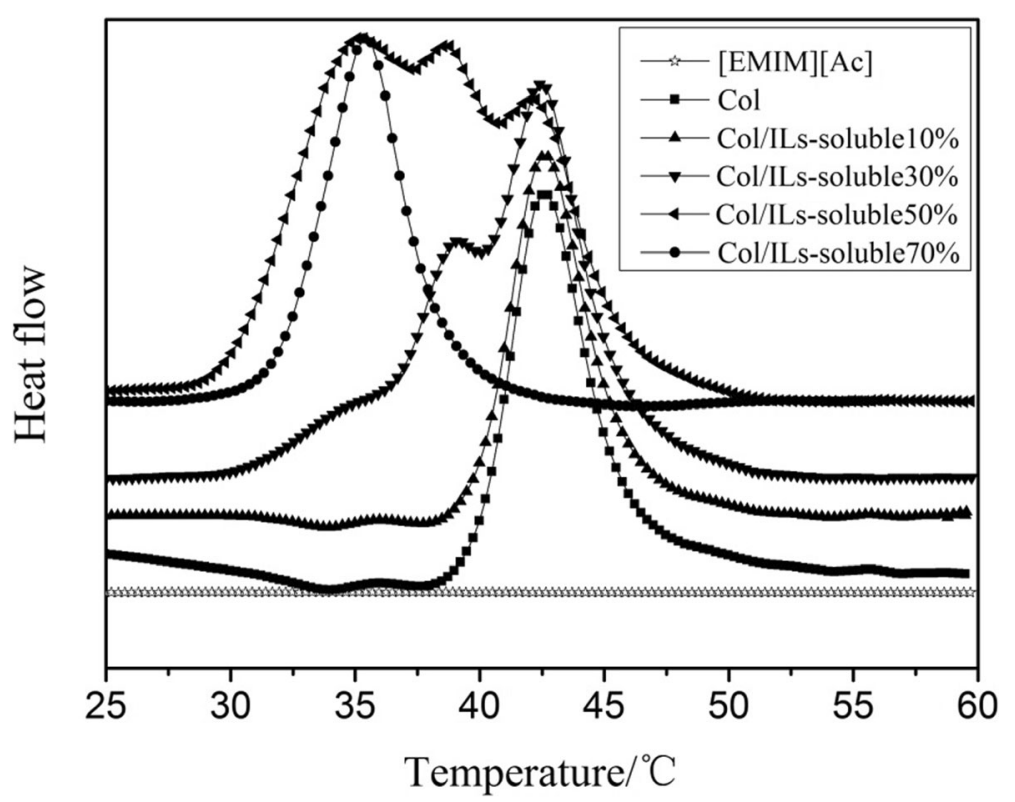

Fig. 6 US-DSC curves of Col, [EMIM][Ac] and collagen samples dissolved in different concentrations of [EMIM][AC]

detected. Together with the results of SDS-PAGE, we can see that although there was no degradation in polypeptide chain during the process of dissolving, the thermal stability of collagen was reduced because of the decreased hydrogen bonds.

\subsection{Fluorescence analysis of soluble collagen}

ANS, which is a fluorescent hydrophobic probe, has high sensitivity to the environment polarity in solution [41]. The introduce of ANS to proteins causing the rise of hydrophobicity is equal to decrease in polarity, leading to the blue shift in maximum peak and an increase in fluorescence intensity [33]. The fluorescence emission spectra of ANS are shown in Fig. 7, displaying four main peaks at $\sim 526.0, \sim 526.8, \sim 529.8$, and $\sim 530.6 \mathrm{~nm}$ in different soluble collagen solutions. With an increase in [EMIM][Ac] concentration, the fluorescence intensity reduced and a red shift resulted from 526.0 to $530.6 \mathrm{~nm}$ in the maximum peak, which indicates the hydrophobicity of the collagen solutions was reduced. Wu et al. [42]

Table 2 Thermodynamic parameters of Col and collagen samples dissolved in different concentrations of [EMIM][AC $]^{a}$

\begin{tabular}{lll}
\hline Samples & $\mathrm{Tm}_{1} /{ }^{\circ} \mathrm{C}$ & $\mathrm{Tm}_{2} /{ }^{\circ} \mathrm{C}$ \\
\hline Col & $35.97 \pm 0.15$ & $42.73 \pm 0.12$ \\
Col/ILs-soluble10\% & $35.68 \pm 0.09$ & $42.47 \pm 0.18$ \\
Col/ILs-soluble30\% & $34.09 \pm 0.17$ & $42.39 \pm 0.10,39.03 \pm 0.14$ \\
Col/ILs-soluble50\% & $29.04 \pm 0.13$ & $42.31 \pm 0.06,38.55 \pm 0.16$, \\
& & $35.36 \pm 0.13$ \\
Col/ILs-soluble70\% & $27.65 \pm 0.11$ & $35.10 \pm 0.13$
\end{tabular}

Values are average \pm standard derivation $(N=3)$ reported that the increase of fluorescence intensity was because of the form of hydrophobic areas from the aggregation of collagen molecules. The results showed that with the increase of [EMIM][Ac] concentration, the polarity of the solutions increased, and the aggregation status of collagen molecules was reduced.

\subsection{DLS analysis of soluble collagen}

DLS has been reported to be a good method to measure the aggregate size [43]. Alterations in the sizes of the soluble collagen aggregates dissolved in different concentrations of [EMIM][Ac] are shown in Fig. 8. The distribution of size regions was bimodal, and comprised two populations of aggregates of $21-122 \mathrm{~nm}$ and 459 $4150 \mathrm{~nm}$, respectively, might attributing to different sizes of collagen aggregates. Differences in intensity and location of these two regions of distribution are also shown in Fig. 8. The two collagen-aggregate distribution regions tended to a smaller size, which demonstrates collagen aggregates with relatively smaller sizes could be more sensitive to the [EMI$\mathrm{M}][\mathrm{Ac}]$ concentration and loosened more easily with an increase in [EMIM][Ac] concentration. The intensity of the first region increased markedly, whereas the second region decreased evidently, which indicates that more small aggregates were produced at a high [EMIM] [Ac] concentration. The average sizes of the collagen aggregates of the Col/ILssoluble $10 \%$, Col/ILs-soluble30\%, Col/ILs-soluble $50 \%$, and Col/ILs-soluble70\% were 1900.81, 1406.46, 983.16, and $334.32 \mathrm{~nm}$, respectively. The size reduction might be caused by disaggregation of collagen aggregates because of the breaking of hydrogen bonds by [EMIM] $[\mathrm{Ac}]$ and an 


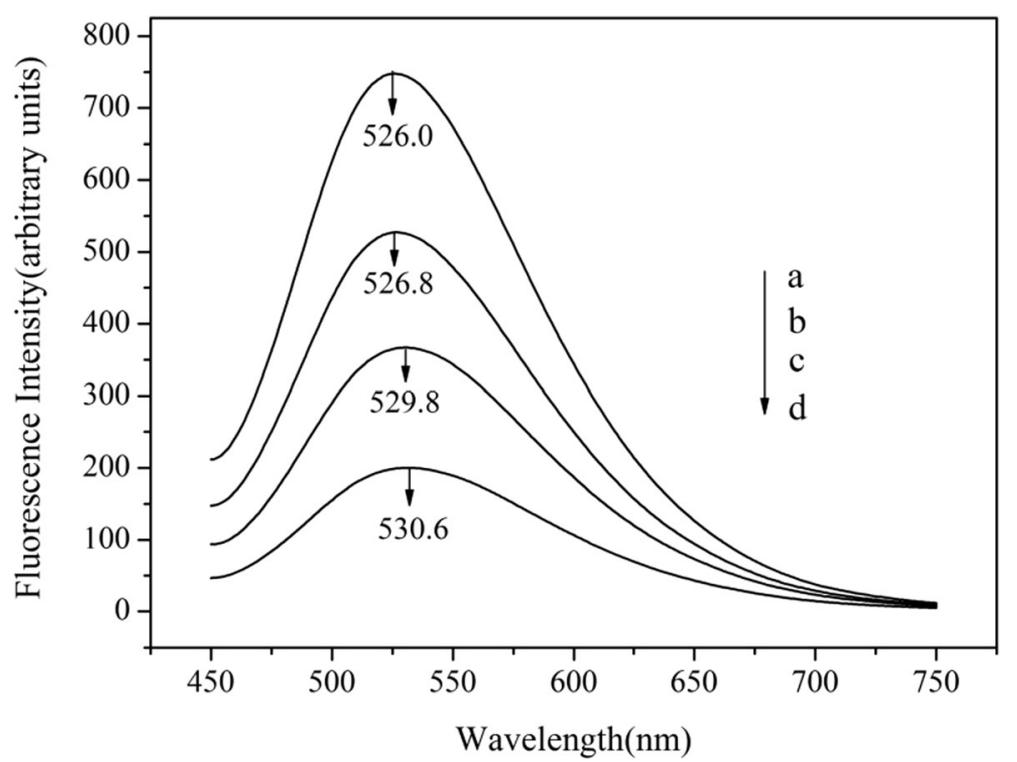

Fig. 7 Fluorescence spectra of ANS in collagen solutions at different [EMIM][Ac] concentrations. a:Col/ILs-soluble10\%, b:Col/Ls-soluble30\%, c:Col/ILs-soluble50\%, d:Col/Ls-soluble70\%

increase of electrostatic repulsion between each collagen chain.

\subsection{AFM images of soluble collagen}

AFM was performed to observe the molecule structures of the soluble collagen. Figure 9 exhibits the morphology changes of Col/ILs-soluble10\%, Col/ILs-soluble30\%, and Col/ILs-soluble50\%, where native collagen was applied as comparison. Characteristic topographies in collagen fibers were observed. By inspection, with increasing [EMI$\mathrm{M}][\mathrm{Ac}]$ concentration, the fiber arrangement tended to be loosened with larger pore sizes, and the fiber length was
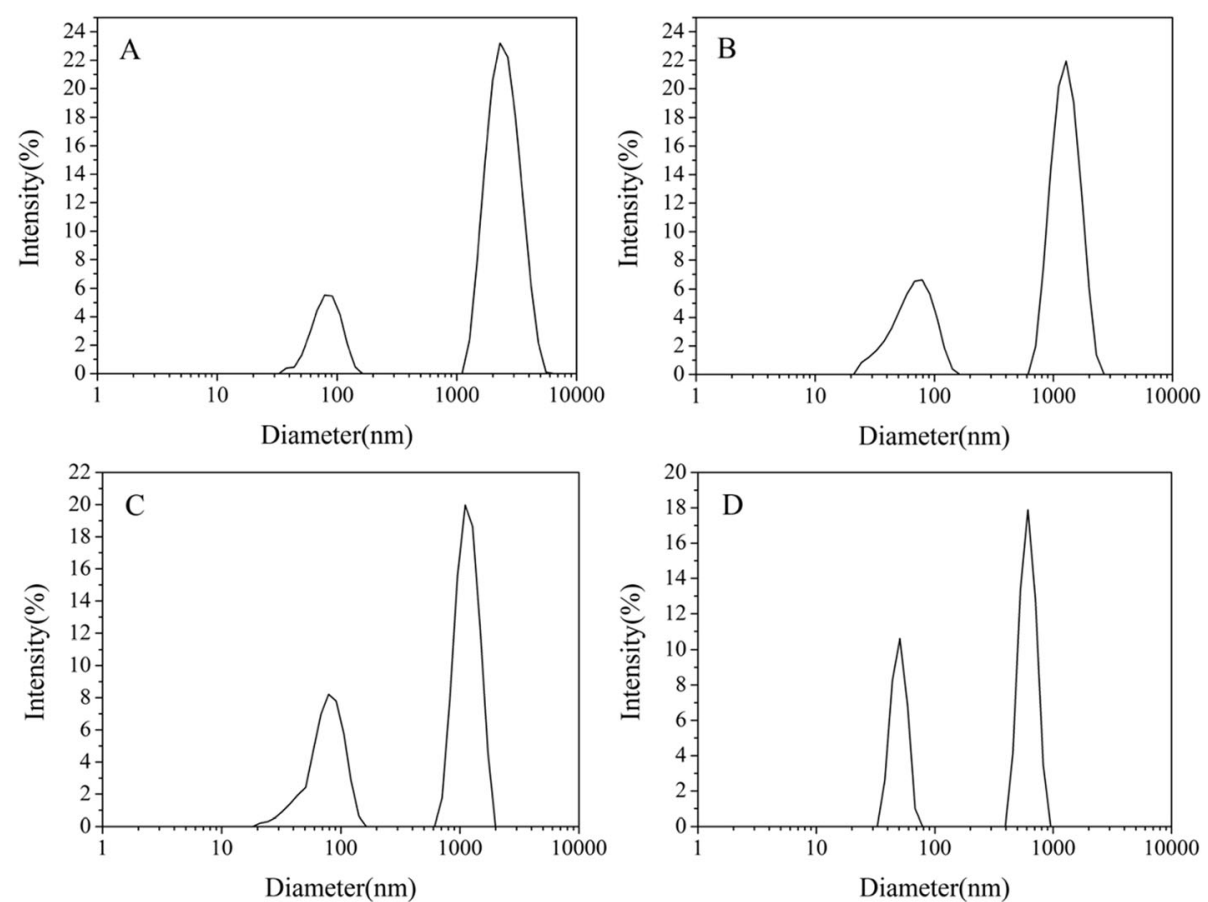

Fig. 8 The sizes and distribution of collagen aggregates determined by DLS dissolved in different concentrations of [EMIM][AC] (a:Col//Lssoluble10\%, b:Col//Ls-soluble30\%, c:Col/ILs-soluble50\%, d:Col/ILs-soluble70\%) 

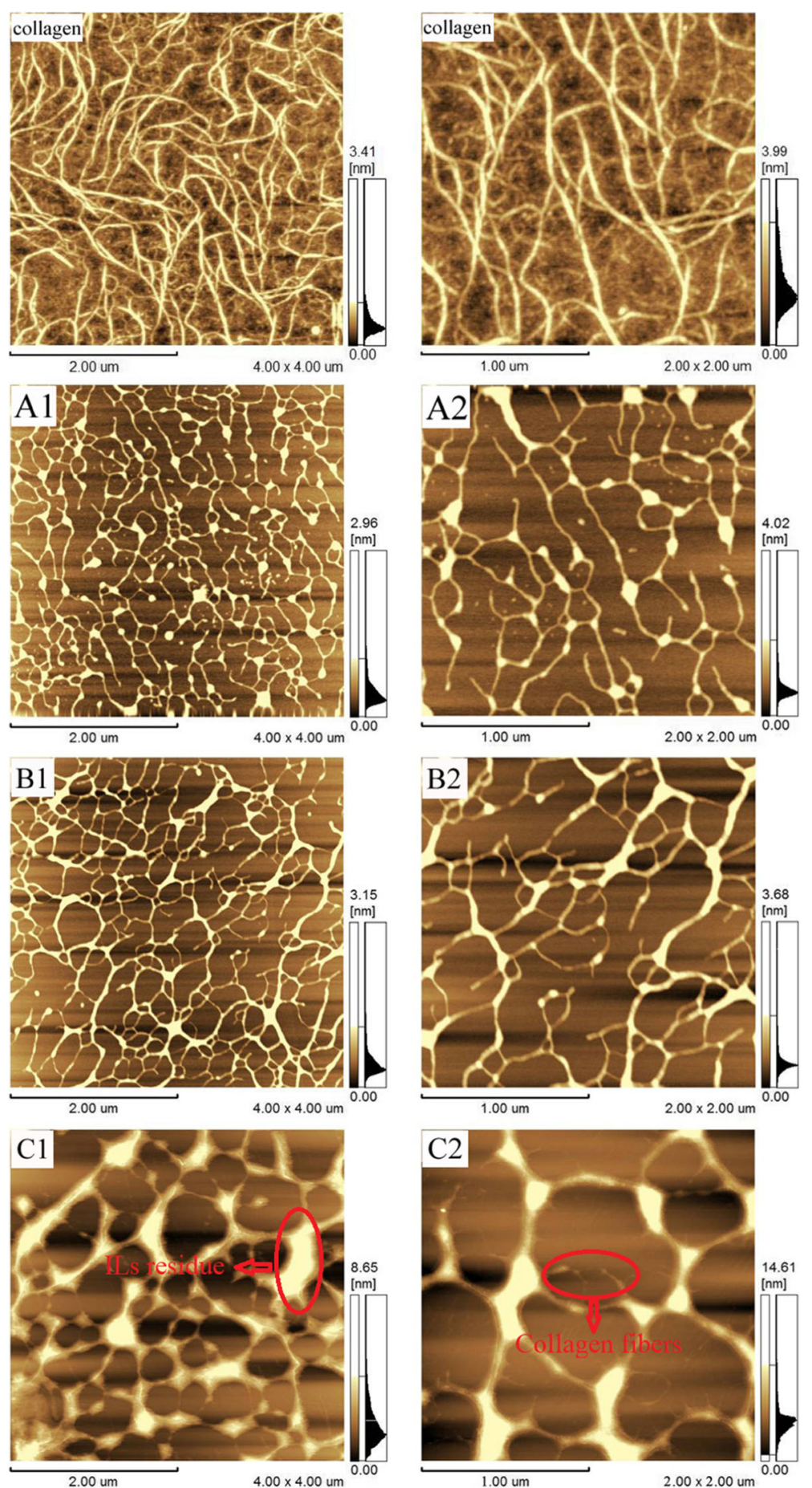

Fig. 9 AFM images of the native collagen and collagen samples dissolved in different concentrations of [EMIM][Ac]. Col/ILs-soluble10\% (A1, A2), Col/ILs-soluble30\% (B1, B2), Col/lLs-soluble50\% (C1, C2), $4.00 \times 4.00 \mu \mathrm{m}(\mathbf{A} 1, \mathbf{B 1}, \mathbf{C 1}), 2.00 \times 2.00 \mu \mathrm{m}$ (A2, B2, C2)

shortened, exhibiting a more sparsely distributed fiber network. This result may indicate that the decreased intermolecular hydrogen bonds caused weakened intermolecular forces between collagen molecules in [EMIM][Ac]. Consequently, the results agreed well with the results for the sizes and distribution of the collagen aggregates in different concentrations of [EMIM][Ac]. Note that the white spots in AFM images were ILs residue, and the thin strings were collagen fibers. Because of the high concentration of [EMIM][Ac], the Col/ILs-soluble70\% sample 
did not yield an AFM image because of ILs residue, although distilled water had been used to wash the sample many times.

\section{Conclusions}

The solubility and dispersion degree of collagen in $70 \%$ [EMIM] $\left[\mathrm{BF}_{4}\right]$ and [EMIM] $[\mathrm{Ac}]$ in concentrations from $10 \%$ to $70 \%$ were studied. Only insoluble collagen fibers were presented from [EMIM] $\left.\mathrm{BF}_{4}\right]$, due to no dissolution of collagen occurred in $[\mathrm{EMIM}]\left[\mathrm{BF}_{4}\right]$. Though dissolved in [EMIM][Ac], the total dissolution of collagen was not achieved and a phenomenon of partial dissolution resulted with the soluble collagen and insoluble collagen fibers across the entire [EMIM][Ac] concentration range. For the insoluble collagen fibers, although the partial interruption of hydrogen bonds did not make it dissolved, the entire fiber arrangement was looser and the thermal stability decreased with the increase of ILs polarity and concentration. For the soluble collagen, no degradation of polypeptide chain occurred during dissolution. Owing to the fact that more hydrogen bonds were broken with the increase of ILs concentration, the reduction in aggregation states of soluble collagen was more prominent, and resulted in a decreased thermal stability, a looser fiber arrangement, and shorter fiber lengths. Therefore, $[\mathrm{EMIM}]\left[\mathrm{BF}_{4}\right]$ is a good dispersion agent for collagen with no destruction to collagen structures, while [EMI$\mathrm{M}][\mathrm{Ac}]$ is a good solvent and dispersion agent for collagen, depending on the concentration. The information obtained may be useful in the application of ILs in more collagen products with a suitable sort and concentration to retain the structural integrity of the collagen.

\begin{abstract}
Abbreviations
[EMIM][Ac]: 1-ethyl-3-methylimidazolium acetate; [EMIM][BF $]$ ]: 1-ethylmethylimidazolium tetrafluoroborate; Col: Collagen; Col//Ls-

fibrils10\%: Insoluble collagen fibrils from 10\% [EMIM][Ac]; Col//Lsfibrils30\%: Insoluble collagen fibrils from 30\% [EMIM][Ac]; Col//Lsfibrils50\%: Insoluble collagen fibrils from 50\% [EMIM][Ac]; Col//Lsfibrils70\%: Insoluble collagen fibrils from 70\% [EMIM][AC]; Col//Lsfibrils70\%B: Insoluble collagen fibrils from 70\% [EMIM][BF $]$; Col//Lssoluble10\%: Soluble collagen in 10\% [EMIM][Ac]; Col/Ls-soluble30\%: Soluble collagen in 30\% [EMIM][Ac]; Col/Ls-soluble50\%: Soluble collagen in 50\% [EMIM][Ac]; Col/ILs-soluble70\%: Soluble collagen in 70\% [EMIM][Ac]; ILs: Ionic liquids
\end{abstract}

\section{Acknowledgments}

Not applicable

\section{Authors' contributions}

QL has equal contribution to the first author. SL designed and performed the experiments, analyzed the data and wrote the manuscript. QL performed the experiments of soluble collagen and was a major contributor in writing the manuscript. GL analyzed the data and improved the manuscript. All authors read and approved the final manuscript.

\section{Funding}

The research is funded by the National Natural Science Foundation of China (Nos. 21776184 and 21476147).

\section{Availability of data and materials}

All data generated or analyzed during this study are included in this article.

Ethics approval and consent to participate

Not applicable

\section{Consent for publication}

Not applicable

\section{Competing interests}

The authors declare that they have no competing interests.

Received: 29 July 2019 Accepted: 11 October 2019

Published online: 11 November 2019

\section{References}

1. Meli L, Miao J, Dordick JS, Linhardt RJ. Electrospinning from room temperature ionic liquids for biopolymer fiber formation. Green Chem. 2010; 12:1883-92.

2. Yuan J, Wang Q, Fan XJ. Dyeing behaviors of ionic liquid treated wool. Appl Polym Sci. 2010;117:2278-83.

3. Ventura SPM, Santos LDF, Saraiva JA, Coutinho JAP. Ionic liquids microemulsions: the key to Candida antarctica lipase B superactivity. Green Chem. 2012;14:1620-5.

4. Xie HB, Li SH, Zhang SB. Ionic liquids as novel solvents for the dissolution and blending of wool keratin fibers. Green Chem. 2005;7:606-8.

5. Duan X, Xu J, He B, Li J, Sun Y. Preparation and rheological properties of cellulose/chitosan homogeneous solution in ionic liquid. BioResources. 2011;6:4640-51.

6. Fukaya Y, Hayashi K, Wada M, Ohno H. Cellulose dissolution with polar ionic liquids under mild conditions: required factors for anions. Green Chem. 2008;10:44-6.

7. Wu Y, Sasaki T, Irie S, Sakurai K. A novel biomass-ionic liquid platform for the utilization of native chitin. Polymer. 2008;49:2321-7.

8. Phillips DM, Drummy LF, Conrady DG, Fox DM. Dissolution and regeneration of Bombyx mori silk fibroin using ionic liquids. J Am Chem Soc. 2004;126:14350-1.

9. Potorac S, Popa M, Picton L, Dulong V, Verestiuc L. Collagen functionalized with unsaturated cyclic anhydrides-interactions in solution and solid state. Biopolymers. 2014;101:228-36.

10. Mehta A, Rao JR, Fathima NN. Effect of ionic liquids on the different hierarchical order of type I collagen. Colloids Surf B. 2014;117:376-82.

11. Lin H, Dan W, Dan NJ. The water state in crosslinked poly (vinyl alcohol)collagen hydrogel and its swelling behavior. Appl Polym Sci. 2012;123:2753-61.

12. Arafat MT, Tronci G, Yin J, Wood DJ, Russell SJ. Biomimetic wet-stable fibres via wet spinning and diacid-based crosslinking of collagen triple helices. Polymer. 2015;77:102-12.

13. Kezwon A, Wojciechowski K. Effect of temperature on surface tension and surface dilational rheology of type I collagen. Colloids Surf A Physicochem Eng Asp. 2014:460:168-75.

14. Fratzl P. Collagen: structure and mechanics, an introduction. Springer US 2008:1-13.

15. Zeugolis DI, Khew ST, Yew ES, Ekaputra AK, Tong YW, Yung LY, Hutmacher DW, Sheppard C, Raghunath M. Electro-spinning of pure collagen nano-fibres - just an expensive way to make gelatin? Biomaterials. 2008;29:2293-305.

16. Wang G, Guo J, Zhuang L, Wang Y, Xu B. Dissolution and regeneration of hide powder/cellulose composite in Gemini imidazolium ionic liquid. Int J Biol Macromol. 2015;76:70-9.

17. Zhang M, Ding $C$, Huang $L$, Chen L, Yang $H$. Interactions of collagen and cellulose in their blends with 1-ethyl-3-methylimidazolium acetate as solvent. Cellulose. 2014;21:3311-22.

18. Boy R, Narayanan G, Chung CC, Kotek R. Novel cellulose-collagen blend biofibers prepared from an amine/salt solvent system. Int J Biol Macromol. 2016:92:1197-204.

19. Muhammad N, Gonfa G, Rahim A, Ahmad P, labal F, Sharif F, Khan AS, Khan FU. Investigation of ionic liquids as a pretreatment solvent for extraction of collagen biopolymer from waste fish scales using COSMO-RS and experiment. Mol Liq. 2017;232:258-64

20. Liu J, Xu Z, Chen Y, Fan H, Shi B. 1-butyl-3-methylimidazolium acetate as an alternative solvent for type I collagen. J Am Leather Chem As. 2014;109: 189-96. 
21. Zheng X, Meng Z, Liu J, Tang K. Dissolution and regeneration of collagen fibers using ionic liquid. Int J Biol Macromol. 2012;51:440-8.

22. Hu Y, Liu L, Dan W, Dan N, Gu ZJ. Evaluation of 1-ethyl-3methylimidazolium acetate based ionic liquid systems as a suitable solvent for collagen. Appl Polym Sci. 2013;130:2245-56.

23. Harada LK, Pereira JFB, Campos WF, Silva EC, Tubino M. Insights into protein-ionic liquid interactions aiming at macromolecule delivery systems. J Braz Chem Soc. 2018;29:1983-98.

24. Tarannum A, Muvva C, Mehta A, Rao JR, Fathima NN. Phosphonium based ionic liquids-stabilizing or destabilizing agents for collagen? RSC Adv. 2016; 6:4022-33.

25. Tarannum A, Jonnalagadda RR, Fathima NN. Stability of collagen in ionic liquids: ion specific Hofmeister series effect. Spectroc Acta Pt A-Molec Biomolec Spectr. 2019;212:343-8.

26. Tarannum A, Adams A, Fathima NN. Impact of ionic liquids on the structure and dynamics of collagen. J Phys Chem B. 2018;122:1060-5.

27. Tarannum A, Rao JR, Fathima NN. Choline based amino acid ILs-collagen interaction: enunciating its role in stabilization/destabilization phenomena. J Phys Chem B. 2018;122:1145-51.

28. Tarannum A, Muvva C, Mehta A, Rao JR, Fathima NN. Role of preferential ions of ammonium ionic liquid in destabilization of collagen. J Phys Chem B. 2016:120:6515-24.

29. Alla JP, Rao JR, Fathima NN. Integrated depilation and fiber opening using aqueous solution of ionic liquid for leather processing. ACS Sustain Chem Eng. 2017;5:8610-8.

30. Zhang ZK, Liu WT, Li GY, Shi B. Physicochemical properties of collagen, gelatin and collagen hydrolysate derived from bovine limed split wastes. J Soc Leather Technol Chem. 2006;90:23.

31. Li ZR, Wang B, Chi CF, Zhang QH, Gong YD, Tang JJ, Luo HY, Ding GF. Isolation and characterization of acid soluble collagens and pepsin soluble collagens from the skin and bone of Spanish mackerel (Scomberomorous niphonius). Food Hydrocoll. 2013;31:103-13.

32. Laemmli UK. Cleavage of structural proteins during the assembly of the head of bacteriophage T4. Nature. 1970;227:680-5.

33. Kamyshny A, Magdassi S, Relkin PJ. Chemically modified human immunoglobulin G: hydrophobicity and surface activity at air/solution Interface. Colloid Interf Sci. 1999:212:74-80.

34. Makhatadze Gl, Privalov PL. Energetics of protein structure. Adv Protein Chem. 1995;47:307-425.

35. Pietrucha K. Changes in denaturation and rheological properties of collagen-hyaluronic acid scaffolds as a result of temperature dependencies. Int J Biol Macromol. 2005;36:299-304.

36. Yan M, Li B, Zhao X, Ren G. Characterization of acid-soluble collagen from the skin of walleye Pollock (Theragra chalcogramma). Food Chem. 2008;107: $1581-6$.

37. Staicu T, Cîrcu V, Ioniță G, Ghica C, Popa VT, Micutz M. Analysis of bimodal thermally-induced denaturation of type I collagen extracted from calfskin. RSC Adv. 2015:5:38391-406.

38. Zhang M, Ding CC, Yang JH, Lin S, Chen LH, Huang LL. Study of interaction between water-soluble collagen and carboxymethyl cellulose in neutral aqueous solution. Carbohydr Polym. 2016;137:410-7.

39. Brodsky B, Persikov AV. Molecular structure of the collagen triple helix. Adv Protein Chem. 2005;70:302-33.

40. Yang H, Li Q, Liu SC, Li GY. Acetic acid/1-ethyl-3-methylimidazolium acetate as a biphasic solvent system for altering the aggregation behavior of collagen molecules. J Mol Liq. 2018;262:78-85.

41. Yuu S, Hiroharu Y. Fluorescence lifetime probe for solvent microviscosity utilizing anilinonaphthalene sulfonate. Anal Chem. 2010;82:5470-6.

42. Wu K, Liu W, Li G. The aggregation behavior of native collagen in dilute solution studied by intrinsic fluorescence and external probing. Spectrochim Acta A. 2013:102:186-93.

43. Politi J, Stefano LD, Longobardi S, Giardina P, Rea I, Methivier C, Pradier CM, Casale S, Spadavecchia J. The amphiphilic hydrophobin Vmh2 plays a key role in one step synthesis of hybrid protein-gold nanoparticles. Colloid Surf B. 2015;136:214-21.

\section{Publisher's Note}

Springer Nature remains neutral with regard to jurisdictional claims in published maps and institutional affiliations.

\section{Submit your manuscript to a SpringerOpen ${ }^{\circ}$ journal and benefit from:}

- Convenient online submission

- Rigorous peer review

- Open access: articles freely available online

- High visibility within the field

- Retaining the copyright to your article

Submit your next manuscript at $\boldsymbol{\nabla}$ springeropen.com 